\title{
REPRESENTATION OF ABSTRACT RIESZ POTENTIALS OF THE ELLIPTIC TYPE
}

\author{
BY A. V. BALAKRISHNAN
}

Communicated by Einar Hille, June 13, 1958

Using semigroup theory we are able to obtain an abstract definition of the Riesz potentials of the elliptic type as closed linear operators, as well as a representation for them without using continuation.

Let

$$
T(\xi), \xi=\left(\xi_{1}, \xi_{2}, \cdots, \xi_{n}\right),-\infty<\xi_{k}<\infty,
$$

be an $n$-parameter group (strongly continuous) of endomorphisms over a $B$-space $X$. Let

$$
T(\xi)=\prod_{k=1}^{n} T_{k}\left(\xi_{k}\right)
$$

each $T_{k}\left(\xi_{k}\right)$ being a strongly continuous one-parameter group with infinitesimal generator $A_{k}$. Let

$$
C=\sum_{i=1}^{n} \sum_{j=1}^{n} a_{i j} A_{i} A_{j},
$$

where the matrix $\left[a_{i j}\right]$ is real symmetric and positive definite. Then $C$ is the infinitesimal generator of a one-parameter strongly continuous semigroup $S(t), 0<t$, and further sup $\|S(t)\|<\infty$. Now, in some previous work the author has shown that in such a case it is possible to define $(-C)^{\alpha}, \operatorname{Re} \alpha>0$, as closed linear operators, interpolating integral powers and having the semigroup property in $\alpha$. Moreover, they have an explicit representation as Bochner integrals in terms of $S(t)$, which for $0<\operatorname{Re} \alpha<1$, is

$$
(-C)^{\alpha} x=\frac{1}{\Gamma(-\alpha)} \int_{0}^{\infty}[S(t) x-x] t^{-\alpha-1} d t
$$

for $x \in D(C)$. Next, to simplify the notation, let

$$
C=\sum_{i=1}^{n} A_{i}^{2}
$$

Then for every $x \in X$,

$$
S(t) x=\frac{1}{\left(2(\pi t)^{1 / 2}\right)^{n}} \int_{E_{n}} T(\xi) x \exp \left[-\sum_{1}^{n} \xi_{k}^{2} / 4 t\right] d \xi_{1} \cdots d \xi_{n} .
$$


The Riesz potentials are obtained by substituting (2) into (1). Thus:

$$
(-C)^{\alpha} x=\frac{4^{\alpha} \Gamma(\alpha+n / 2)}{\pi^{n / 2} \Gamma(-\alpha)} \int_{E_{n}^{+}}[T(\xi) x+T(-\xi) x-2 x]|\xi|^{-2 \alpha-n} d V_{\xi}
$$

where

$$
\begin{aligned}
& E_{n} \text { is the } 2^{n} \text {-ant in which } \xi_{k} \geqq 0 \text { for all } k, \\
& |\xi|=\left[\xi_{1}^{2}+\cdots+\xi_{n}^{2}\right]^{1 / 2}, \\
& d V_{\xi} \text { is the } n \text {-dimensional volume element. }
\end{aligned}
$$

With slight modification it is possible to write this as:

$$
\begin{aligned}
(-C)^{\alpha} x= & \frac{4^{\alpha} \Gamma(\alpha+n / 2)}{\pi^{n / 2} \Gamma(-\alpha)(n+2 \alpha-1)} \int_{E_{n}^{+}}[T(\xi)-T(-\xi)] \\
& \cdot\left[\sum_{1}^{n} \xi_{k} A_{k} x\right]|\xi|^{-2 \alpha-n} d V_{\xi},
\end{aligned}
$$

where the integral is to be taken in the Cauchy sense at infinity. In this latter form, for $\alpha=1 / 2$ for instance, we get an abstract version of the conjugate transform in $L_{p}\left(E_{n}\right)$ spaces in its usual representation.

University of Southern California 